\title{
IMPACT OF SPEED SINTERING ON MARGINAL DISCREPANCY, INTERNAL FIT AND MICROLEAKAGE OF MONOLITHIC ZIRCONIA CROWNS WITH DIFFERENT CHAMFER WIDTH
}

\author{
Samah Saker*, Dalia Zahran** and Walid Al-Zordk***
}

\begin{abstract}
Purpose: The current in-vitro investigation analyzed the impact of speed sintering and different finish line width on marginal adaptation, internal fit and microleakage of translucent monolithic zirconia crowns.

Materials and Methods: Sixty maxillary premolars were reduced based on basic guidelines of tooth preparation for a zirconia crown with either $0.5,0.8$ and $1.2 \mathrm{~mm}$ chamfer width $(\mathrm{n}=2 \emptyset \mathrm{each}$ finish line width). After digital scanning and milling of zirconia crowns, the sintering procedure was performed in special furnace for two different sintering programs ( $\mathrm{n}=10$ per group) following either standard or speed sintering procedure. Each crown was cemented to its corresponding prepared tooth utilizing self-adhesive-resin cement. After 15000 thermal cycles between $5^{\circ} \mathrm{C}$ and $55^{\circ} \mathrm{C}$, specimens were submersed in methylene blue for 12 hours. After sectioning buccolingually, marginal gap and cement film thickness values were recorded at nine sites using a digital microscope followed by microleakage scores examination.
\end{abstract}

Results: Tested groups with $0.5 \mathrm{~mm}$ chamfer widths had the greatest mean marginal gap values of $47.9 \pm 5.4$ and $50.1 \pm 5.6 \mu \mathrm{m}$ for both conventional and speed sintering respectively, while groups with 1.2 chamfer widths had the lowest mean gap values of $42.3 \pm 5.6$ and $44.5 \pm 5 \mu \mathrm{m}$ for both standard and speed sintering, respectively. Two-way ANOVA revealed statically insignificant impact of sintering procedure on crown adaptation $(p=.08)$. The greatest mean microleakage score $(3 \pm 088 \mu \mathrm{m})$ was obtained from the $0.5 \mathrm{~mm}$ chamfer width group, while the smallest $(2 \pm 0.78 \mu \mathrm{m})$ score was obtained from the $1.2 \mathrm{~mm}$ chamfer width group.

Conclusions: Marginal adaptation and internal fit of translucent monolithic zirconia crowns are affected by width of the preparation margin while sintering protocol has little impact on adaptation.

KEY WORD: finish line width, translucent zirconia, sintering program.

\footnotetext{
* Associate Professor, Fixed prosthodontics Department, Faculty of Dentistry, Mansoura University, Mansoura, Egypt. ** Associate Professor of Oral Biology Department, Faculty of Dentistry, Tanta University, Tanta, Egypt.

***Assistant Professor, Fixed Prosthodontics Department, Faculty of Dentistry, Mansoura University, Mansoura, Egypt.
} 


\section{INTRODUCTION}

With the presentation of monolithic zirconia restorations, drawbacks identified with the clinical utilization of conventional zirconia restorations has been disposed of, that is, the need to veneer the coping with feldspathic porcelain. ${ }^{1-3}$ Recently, high translucent zirconia has been introduced in an attempt to enhance the esthetic outcomes. Compared with conventional zirconia, high translucent zirconia ceramic has different mechanical and optical behaviors, and recommended for monolithic restorations with restricted application and conservative tooth preparation. ${ }^{3}$

Processing of current dental zirconia is grouped into three phases, green, pre-sintered and fully sintered. The structures got from the green and presintered zirconia blocks are processed in greater extents to neutralize any potential volumetric shrinkage $(20 \%$ to $25 \%)$ that pursues the last sintering stage..$^{4-6}$ The snappier processing of these $\mathrm{ZrO}_{2}$ blocks makes the wear of the equipment be lower than that in blocks processed in the completely sintered stage. As a matter of fact, post-milling dimensional changes like shrinkage don't happen in completely sintered zirconia; be that as it may, its procedure is very unique and tedious because of its high hardness..$^{7-9}$

Among manufacturers, the sintering temperature of zirconia generally ranges between $1400^{\circ} \mathrm{C}$ to $1600^{\circ} \mathrm{C}$. The final sintering temperature and duration adjusts the grain size of zirconia material. The higher the sintering temperature and the more extended duration, the bigger the grain size. As the grain estimate increments $>1 \mu \mathrm{m}$, zirconia turns out to be less steady and more inclined to higher stage change (tetragonal to monoclinic stage). Then again, lower crack strength can be acquired from littler grain measure $<0.2 \mu \mathrm{m}$. The most well-known sintering strategy for zirconia utilizes customary heaters at temperatures somewhere in the range of $1350^{\circ} \mathrm{C}$ and $1600^{\circ} \mathrm{C}$ and holding times extending from 2 to 4 hours. ${ }^{10-15}$
A fast sintering procedure was introduced by manufacturers as a substitute to conventional sintering procedure that is proposed to be more economic and saving time. Producers" "in-house" testing assume comparable optical properties and adaptation of zirconia restorations with either conventional or speed sintering procedures. However, there is no sufficient data to support this claim. A few in vitro investigations have recently pointed out that, processing of zirconia restoration at high sintering temperatures accompanied with short sintering durations increased the flexural strength of zirconia without affecting its color and translucency..$^{16}$ In addition; an in vitro investigation has been conducted recently declared that, the sintering durations does not have an impact on the marginal discrepancy of zirconia copings. ${ }^{17}$

The dissolution of the luting agent and subsequent microleakage is associated with the increased marginal gap of the restoration. ${ }^{18-20}$ Microleakage is a major causative factor for pulpal inflammation, pulp necrosis and potentially necessitate endodontic intervention. ${ }^{21-24}$ Marginal misfit accelerates plaque accumulation and modifies the composition of the subgingival microflora, participating to the initiation and development of periodontal diseases. Microleakage may be described as the passage of fluids, bacteria, ions or molecules between the tooth substrate and the restorative material. ${ }^{23-28}$ Marginal discoloration, postoperative sensitivity and recurrent caries are reported as consequent complications of microleakage. ${ }^{29}$ It was declared that the estimated annual complication rate for single unit zirconia crowns was $0.09 \%$ for secondary caries and $0 \%$ for marginal staining. ${ }^{30}$

Few studies address the dimensional alterations stem from the sintering procedure and the effect of such alterations on the fit of zirconia crowns. ${ }^{16,31}$ Accordingly, the aim of the current study was to explore the effect of different chamfer finish line widths $(0.5,0.8,1 \mathrm{~mm})$ on the marginal fit, internal adaptation and microleakage of translucent monolithic zirconia crowns manufactured by computer- 
aided design/computer-aided manufacturing (CAD/ CAM) using standard and speed sintering protocols. Thus, the null hypotheses of this study were there is no significant effect of finish line width, and speed sintering procedure on marginal fit, internal adaptation and microleakage of translucent monolithic zirconia crowns.

\section{MATERIALS AND METHODS}

Sixty intact, defect-free human maxillary premolar teeth extracted for periodontal reasons, with approximately similar coronal dimensions were sampled in the current investigation. All the teeth had been collected in the Oral and Maxillofacial Surgery Department, Faculty of Dentistry, Mansoura University. The teeth were debrided, cleaned and inspected under magnification to detect any cracks. The selected teeth were stored in $0.1 \%$ thymol solution at room temperature.

All teeth were individually and vertically fixed in auto-polymerizing acrylic resin (Acrostone, Acrostone Dental Manufacture, Egypt) to a level 2 $\mathrm{mm}$ apical to the cemento-enamel junction. For each tooth, a pre-preparation silicon index (Ghenesyl, Lascod, Italy) was fabricated and sectioned buccolingually to verify tooth reduction and check the contour of the final restoration. Tooth reduction was performed using high-speed diamond (öko DENT, Germany). To control the tooth preparation, milling unit (Milling unit BF 2, bredent GmbH Co, Senden, Germany) was used to compete the tooth reduction. 0.5, 0.8 and 1.2-millimeter wide individual chamfer preparation ( $\mathrm{n}=20$ per each chamfer width). The final preparations had the following criteria; the preparation margin located $1 \mathrm{~mm}$ above cementoenamel junction, $2 \mathrm{~mm}$ occlusal reduction and 8-degree convergence angle. The preparations were rounded and smoothened, except for the gingival margins. The sectioned pre-preparation index was used to assess the finished preparation. All preparations were performed by single operator.
For each prepared tooth, impression was made using polyvinyl siloxane impression material (Ghenesyl, Lascod, Italy) and poured with type IV dental stone (SHERA PREMIUM, SHERA Werkstoff-Technologie GmbH, Germany) following manufacturer's instructions. After complete setting of the stone die, it was fixed on the scan base of the optical scanner (Ceramill Map400, Amann Girrbach $\mathrm{GmbH}$, Germany) and the scanning procedure was completed. Each zirconia crown was designed using CAD-CAM design software (Ceramill Mind CAD version 3.5.6.1408, Amann Girrbach GmbH). The job definition included determination of tooth number, restoration type and material. Certain parameters were determined such as defining of the margin, cement gap thickness $(50 \mu \mathrm{m})$ with $1 \mathrm{~mm}$ from the margin and restoration thickness. After calculation of the tool path, the designed crown was sent to the milling machine (Ceramill Motion 25 axis, Amann Girrbach GmbH). All crowns were milled from pre-sintered zirconia blocks (Zolid FX Preshaded, Amann Girrbach GmbH).

Before sintering, the sintering parameters for zirconia were set on the furnace's control panel following either standard sintering (SS) or fast sintering (FS) procedures following manufacturer's instructions. For each group of chamfer width $(n=20)$, half of the crowns $(n=10)$ were sintered following conventional sintering program and the other half of crowns $(n=10)$ were sintered following speed sintering program (Table 1). All specimens were sintered using zirconia sintering furnace (Ceramill Therm S, Amann Girbach AG). After sintering, all zirconia crowns were cleaned and the dimensions of each crown were checked with digital caliper sensitive to $0.01 \mathrm{~mm}$ for standardization. Finally, all zirconia crowns were subjected to glaze cycles in a porcelain furnace (Prgramat P300, Ivoclar Vivadent) following manufacturer's recommendations. Each crown was checked on its respective tooth for seating without interferences and the margin was evaluated with dental explorer and $2.5 \mathrm{x}$ magnification loop. 
TABLE (1) Sintering parameters for standard and speed sintering procedures of tested zirconia.

\begin{tabular}{|c|c|c|c|c|c|c|c|c|c|}
\hline $\begin{array}{c}1^{\text {st }} \text { ramp } \\
\quad \text { up }\end{array}$ & Temp & Hold & $2^{\text {nd }}$ ramp up & Temp & Hold & $1^{\text {st }}$ ramp down & Temp & $2^{\text {nd }}$ ramp down & Temp \\
\hline \multicolumn{10}{|c|}{ Standard sintering } \\
\hline $20^{\circ} \mathrm{C} / \mathrm{min}$ & $900^{\circ} \mathrm{C}$ & na* & $10^{\circ} \mathrm{Cmin}$ & $1450^{\circ} \mathrm{C}$ & $120 \mathrm{~min}$ & $20^{\circ} \mathrm{Cmin}$ & $200^{\circ} \mathrm{C}$ & na* & na* \\
\hline \multicolumn{10}{|c|}{ Speed sintering } \\
\hline $60^{\circ} \mathrm{C} / \mathrm{min}$ & $990^{\circ} \mathrm{C}$ & na* & $13^{\circ} \mathrm{Cmin}$ & $1450^{\circ} \mathrm{C}$ & $60 \mathrm{~min}$ & na* & $900^{\circ} \mathrm{C}$ & na* & $200^{\circ} \mathrm{C}$ \\
\hline
\end{tabular}

For each zirconia crown, the intaglio surface was air-borne particle abraded with $50 \mu \mathrm{m} \mathrm{Al}_{2} \mathrm{O}_{3}$ according to manufacturer's instructions. Selfadhesive resin cement (Maxcem Elite, Kerr, USA) was used for cementation of each zirconia crown to its respective prepared tooth under constant axial load. After cementation, the margins were finished and polished. Then, all specimens were stored in distilled water for one week. All specimens were subjected to thermal cycling (Thermocycler, SD Mechatronik, Germany) for 15000 cycles which represent two years of clinical service at temperatures alternating between $5^{\circ} \mathrm{C}$ and $55^{\circ} \mathrm{C}$. The dowelling time in each path was 30 seconds.

After artificial aging, two layers of nail varnish were applied except for $1 \mathrm{~mm}$ around the restoration margins. All specimens were immersed in $2 \%$ methylene blue for 12 hours. Then, each tooth was sectioned centrally from buccal to palatal using precision cutting machine (Isomet 400). Subsequently, the sectioned surfaces were prepared with silicon abrasive papers, then cleaned to facilitate the microscopic evaluations.

Misfit (marginal and internal) was assessed microscopically on the sectioned surfaces of the specimens. Each of the sectioned specimen halves, labeled as A and B, were examined with stereomicroscope (Sterioscopic Zoom Microscope, Nikon Corp., Japan) coupled with digital camera.
Digital images of the sliced samples were recorded and stored digitally. Images were analyzed using an imaging data program (Camera DS-LS). For each tooth section, the marginal and internal adaptation between the fitting surface of the crown and the prepared tooth surfaces were measured at 40x at nine predetermined sites in micrometers $(\mu \mathrm{m})$, as follow: buccal margin, mid-buccal chamfer, midbuccal axial, buccal cusp tip, central groove, palatal cusp tip, mid-palatal axial, mid-palatal chamfer and palatal margin (figure 1). The mean of the two halves for each sliced sample was taken as the final measurement for each coping.

After recording of misfit values, the sections were examined using a stereomicroscope to observe the dye penetration of the whole cross section at 10x magnification (figure 2). The depths of dye penetration were scored, ${ }^{22}$ as follow:

0: No microleakage observed.

1: One-third of the chamfer preparation width.

2: Two-third of the chamfer preparation width.

3: All of the chamfer preparation width.

4: More than one-third of the axial wall

5: More than two-third of the axial wall

6: All of the axial walls, including the occlusal edge

7: Exceeding the occlusal edge

Representative specimens were selected for observation by Scanning Electron Microscope (SEM). Each specimen was mounted on stubs and the sectioned surfaces were gold sputter coated to render 
the surface electrically conductive. Specimens were then examined with an environmental scanning electron microscope (JSM-5200, JEOL, Kyoto, Japan) (Figures 2, 3, 4,5 and 6).

\section{Statistical Analysis}

Shapiro-Wilk's test and Levene's test were used to confirm the assumption of normal distribution of the marginal discrepancy and internal fit data, therefore, parametric statistics were used. Means and standard deviations (SD) were calculated for

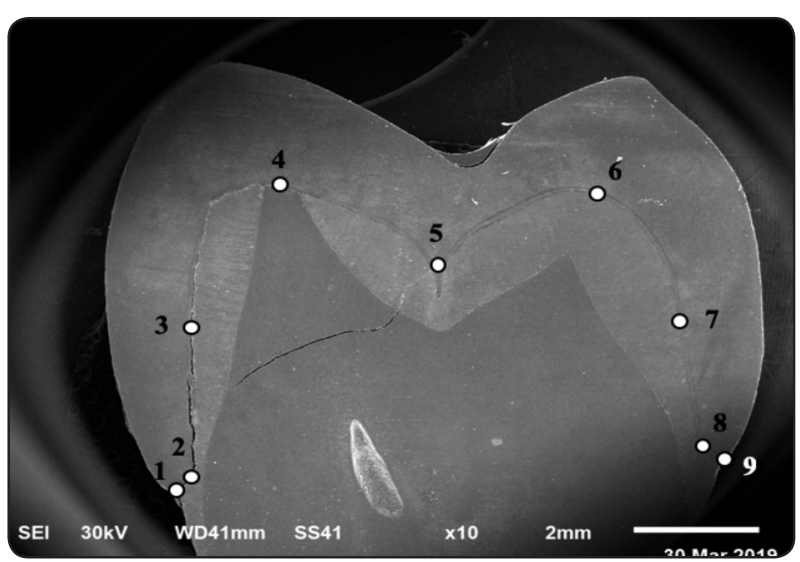

Fig. (1) SEM micrograph of the sectioned surface showing the location of 1-9 points for measuring the marginal and internal adaptation. 1=buccal margin, 2=midbuccal chamfer, $3=$ mid- buccal axial, $4=$ buccal cusp tip, $5=$ central groove, $6=$ palatal cusp tip, $7=$ mid-palatal axial, $8=$ mid-palatal chamfer and $9=$ palatal margin .

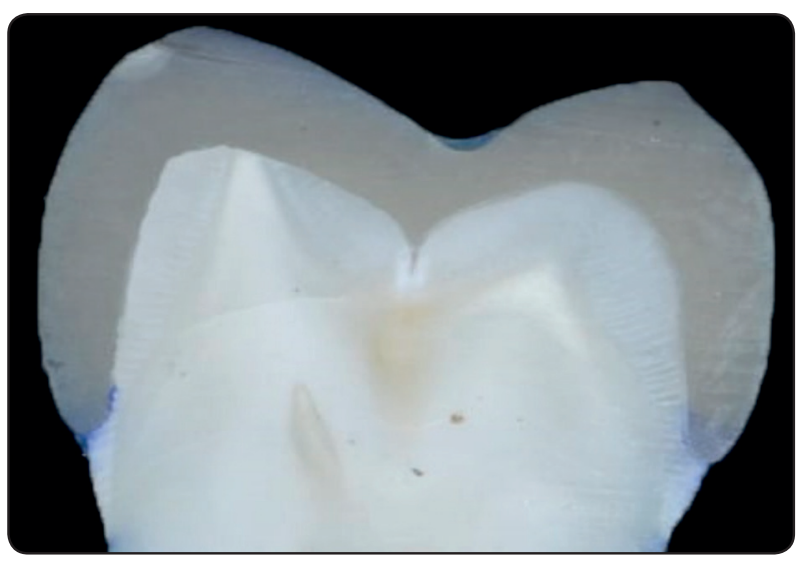

Fig. (2) Representative microscopic view of cross-sectioned specimen showing Score 3 microleakage ( x10). marginal discrepancies and internal fit. A two-way analysis of variance (ANOVA) was used to evaluate the overall statistical significance of differences among the groups under study. Multiple comparisons were made using Tukey`s post-hoc test. $p<0.05$ were considered to be statistically significant in all tests. The microleakage scores were analyzed with the Chi-square and Kruskal-Wallis tests to disclose the differences between the tested groups $(p<0.05)$. All statistical analysis was conducted using SPSS 20.0 software for Windows (SPSS Inc., Chicago, IL, USA).

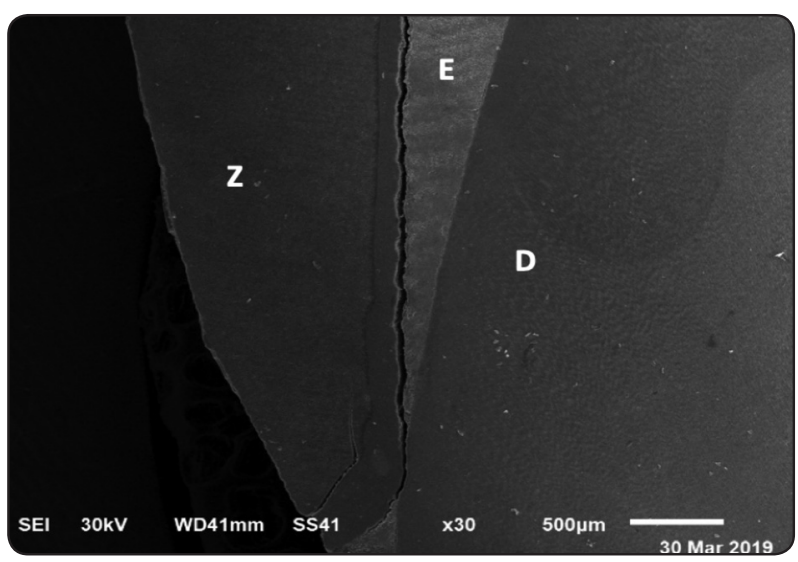

Fig. (3) SEM micrograph of marginal interface between enamel (E) and Dentin, (D), and zirconia restoration (Z) (group $0.5 \mathrm{~mm}$ chamfer width).

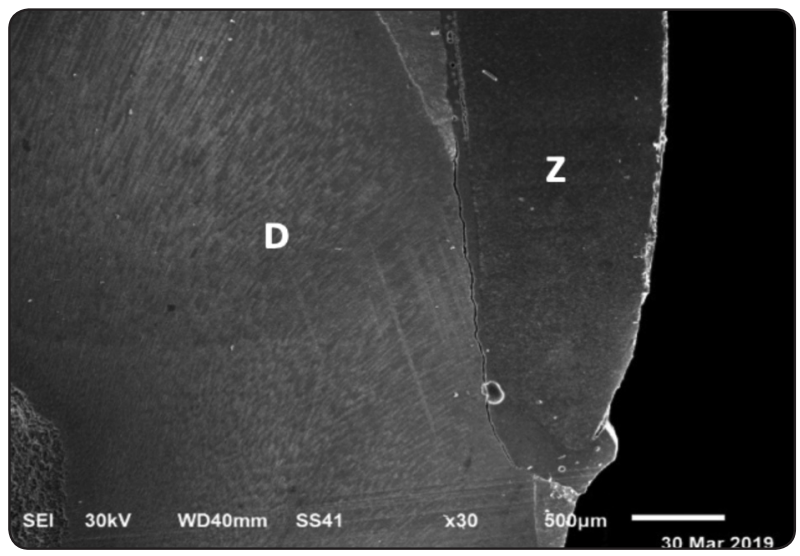

Fig. (4) SEM micrograph of marginal interface between dentin (D) and zirconia restoration (Z), (group 0.8 chamfer width). 


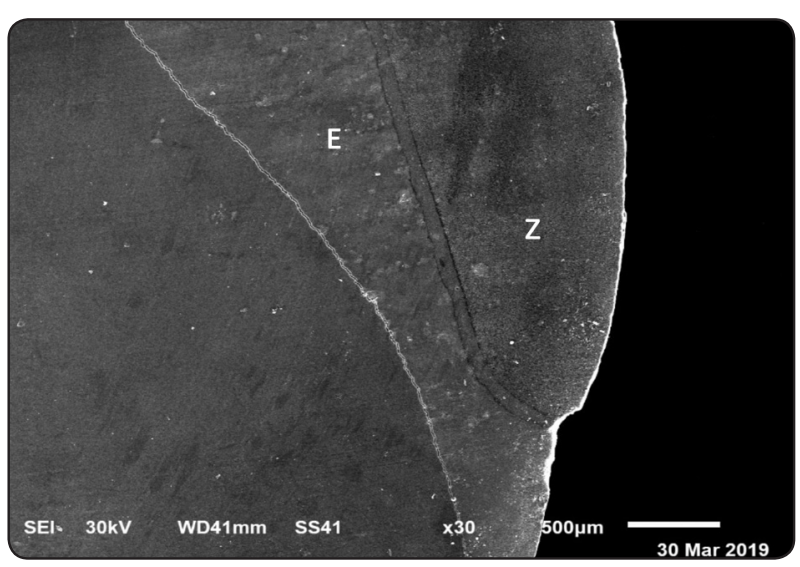

Fig. (5) SEM micrograph of marginal interface between enamel (E) and zirconia restoration (Z), (group $1.2 \mathrm{~mm}$ chamfer width).

\section{RESULTS}

Means and standard deviations of marginal discrepancy and internal adaptation measurements are shown in Table 2. Crowns fabricated from translucent zirconia using either conventional or speed sintering technology yielded a comparable fit. Tested groups with 0.5 chamfer widths had the greatest mean marginal gap values of $47.9 \pm 5.4$ and $50.1 \pm 5.6 \mu \mathrm{m}$ and for both conventional and speed sintering respectively while groups with 1.2 chamfer width had the lowest mean gap values of $42.3 \pm 5.6$ and $44.5 \pm 5 \mu \mathrm{m}$ for both conventional and speed sintering respectively. Two-way ANOVA test revealed statistical significant differences among chamfer width for both marginal discrepancy $(p=0.04)$ and internal adaptation $(p=0.01)$ regardless sintering time used. Statistical analysis revealed that, lowest internal adaptation values recorded

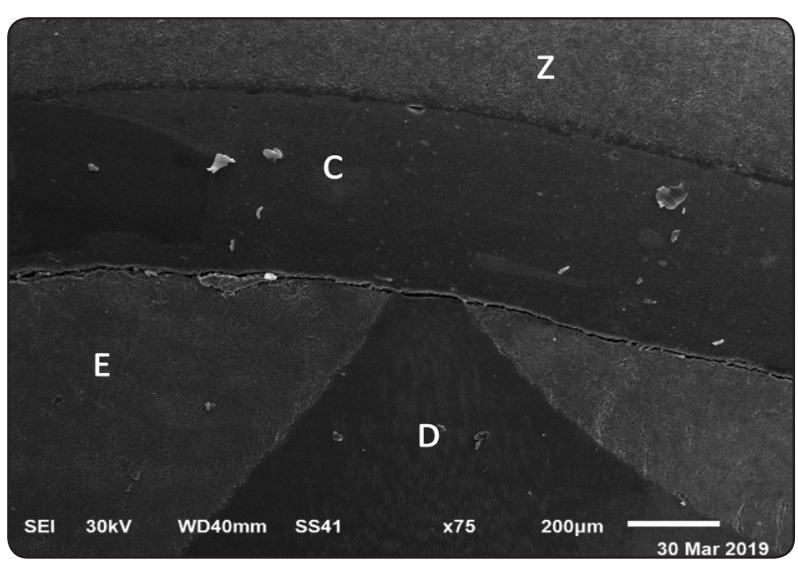

Fig. (6) SEM micrograph showing increased cement film thickness $(\mathrm{C})$ at the occlusal surface. $\mathrm{E}=$ enamel, $\mathrm{D}=$ dentin, $\mathrm{Z}=$ Zirconia restoration

for occlusal surfaces, while the mid axial internal adaptation showed the highest values in all tested groups (table 2). No statistical significant difference between internal adaptation values of the tested groups $(p=0.079)$.

Descriptive statistics of the of microleakage scores for the sintering times and the three chamfer widths used in this, dye penetration data are presented in Table 3. No significant differences in microleakage scores between conventional and speed sintering techniques regardless the chamfer width used ( $p=0.08$ ) according to the Kruskal-Wallis and Chi Square analyses. The greatest mean microleakage score $(3 \pm 0.88)$ was obtained from the $0.5 \mathrm{~mm}$ chamfer width group, while the smallest $(2 \pm 0.78)$ score was obtained from the $1.2 \mathrm{~mm}$ chamfer width group. 
TABLE (2) Means and standard deviations of marginal and internal fit values $(\mu \mathrm{m})$ at nine predetermined sites of conventional and speed sintered monolithic zirconia crowns with different marginal widths.

\begin{tabular}{|c|c|c|c|c|c|}
\hline & Sintering & $\begin{array}{c}\text { Chamfer } \\
\text { width }\end{array}$ & Mean & $\begin{array}{c}\text { Std. } \\
\text { Deviation }\end{array}$ & $\mathrm{N}$ \\
\hline \multirow{6}{*}{ 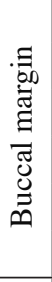 } & \multirow{3}{*}{ Conventional } & $0.5 \mathrm{~mm}$ & 47.64 & 5.2 & 10 \\
\hline & & $0.8 \mathrm{~mm}$ & 46.72 & 4.0 & 10 \\
\hline & & $1.2 \mathrm{~mm}$ & 42.34 & 4.1 & 10 \\
\hline & \multirow{3}{*}{ Speed } & $0.5 \mathrm{~mm}$ & 50.1 & 5.4 & 10 \\
\hline & & $0.8 \mathrm{~mm}$ & 49.6 & 4.3 & 10 \\
\hline & & $1.2 \mathrm{~mm}$ & 44.2 & 5.9 & 10 \\
\hline \multirow{6}{*}{ 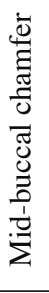 } & \multirow{3}{*}{ Conventional } & $0.5 \mathrm{~mm}$ & 75.3 & 6.8 & 10 \\
\hline & & $0.8 \mathrm{~mm}$ & 76.6 & 4.9 & 10 \\
\hline & & $1.2 \mathrm{~mm}$ & 71.4 & 6.4 & 10 \\
\hline & \multirow{3}{*}{ Speed } & $0.5 \mathrm{~mm}$ & 73.1 & 4.9 & 10 \\
\hline & & $0.8 \mathrm{~mm}$ & 72.1 & 3.77 & 10 \\
\hline & & $1.2 \mathrm{~mm}$ & 71. & 4.5 & 10 \\
\hline \multirow{6}{*}{ 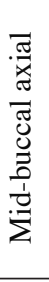 } & \multirow{3}{*}{ Conventional } & $0.5 \mathrm{~mm}$ & 44.9 & 4.7 & 10 \\
\hline & & $0.8 \mathrm{~mm}$ & 40.8 & 3.8 & 10 \\
\hline & & $1.2 \mathrm{~mm}$ & 41.3 & 4.5 & 10 \\
\hline & \multirow{3}{*}{ Speed } & $0.5 \mathrm{~mm}$ & 50.1 & 5.4 & 10 \\
\hline & & $0.8 \mathrm{~mm}$ & 45.4 & 5.9 & 10 \\
\hline & & $1.2 \mathrm{~mm}$ & 40. & 3.6 & 10 \\
\hline \multirow{6}{*}{ 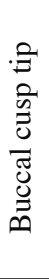 } & \multirow{3}{*}{ Conventional } & $0.5 \mathrm{~mm}$ & 75.3 & 5.9 & 10 \\
\hline & & $0.8 \mathrm{~mm}$ & 77.5 & 6.9 & 10 \\
\hline & & $1.2 \mathrm{~mm}$ & 76.8 & 4.3 & 10 \\
\hline & \multirow{3}{*}{ Speed } & $0.5 \mathrm{~mm}$ & 72.4 & 2.5 & 10 \\
\hline & & $0.8 \mathrm{~mm}$ & 74. & 10.0 & 10 \\
\hline & & $1.2 \mathrm{~mm}$ & 78.8 & 3.3 & 10 \\
\hline
\end{tabular}

\begin{tabular}{|c|c|c|c|c|c|}
\hline \multirow{6}{*}{ 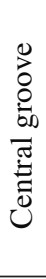 } & \multirow{3}{*}{ Conventional } & $0.5 \mathrm{~mm}$ & 114.2 & 10.8 & 10 \\
\hline & & $0.8 \mathrm{~mm}$ & 117 & 7.6 & 10 \\
\hline & & $1.2 \mathrm{~mm}$ & 106.4 & 7.3 & 10 \\
\hline & \multirow{3}{*}{ Speed } & $0.5 \mathrm{~mm}$ & 115 & 5.6 & 10 \\
\hline & & $0.8 \mathrm{~mm}$ & 111. & 9.4 & 10 \\
\hline & & $1.2 \mathrm{~mm}$ & 114.6 & 5.9 & 10 \\
\hline \multirow{6}{*}{ 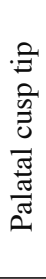 } & \multirow{3}{*}{ Conventional } & $0.5 \mathrm{~mm}$ & 79.6 & 8.7 & 10 \\
\hline & & $0.8 \mathrm{~mm}$ & 81.4 & 5.5 & 10 \\
\hline & & $1.2 \mathrm{~mm}$ & 78.8 & 5.0 & 10 \\
\hline & \multirow{3}{*}{ Speed } & $0.5 \mathrm{~mm}$ & 75.5 & 5.5 & 10 \\
\hline & & $0.8 \mathrm{~mm}$ & 73.9 & 9.9 & 10 \\
\hline & & $1.2 \mathrm{~mm}$ & 81.6 & 8.7 & 10 \\
\hline \multirow{6}{*}{ 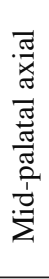 } & \multirow{3}{*}{ Conventional } & $0.5 \mathrm{~mm}$ & 44.7 & 3.6 & 10 \\
\hline & & $0.8 \mathrm{~mm}$ & 41.2 & 3.6 & 10 \\
\hline & & $1.2 \mathrm{~mm}$ & 42.3 & 3.9 & 10 \\
\hline & \multirow{3}{*}{ Speed } & $0.5 \mathrm{~mm}$ & 49.6 & 5.1 & 10 \\
\hline & & $0.8 \mathrm{~mm}$ & 46.2 & 5.5 & 10 \\
\hline & & $1.2 \mathrm{~mm}$ & 41.1 & 3.4 & 10 \\
\hline \multirow{6}{*}{ 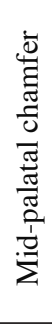 } & \multirow{3}{*}{ Conventional } & $0.5 \mathrm{~mm}$ & 75.2 & 7.2 & 10 \\
\hline & & $0.8 \mathrm{~mm}$ & 76.2 & 4.0 & 10 \\
\hline & & $1.2 \mathrm{~mm}$ & 72.2 & 5.5 & 10 \\
\hline & \multirow{3}{*}{ Speed } & $0.5 \mathrm{~mm}$ & 73.7 & 5.0 & 10 \\
\hline & & $0.8 \mathrm{~mm}$ & 72.7 & 3.3 & 10 \\
\hline & & $1.2 \mathrm{~mm}$ & 71.5 & 4.5 & 10 \\
\hline \multirow{6}{*}{ 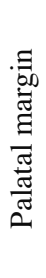 } & \multirow{3}{*}{ Conventional } & $0.5 \mathrm{~mm}$ & 48.1 & 5.5 & 10 \\
\hline & & $0.8 \mathrm{~mm}$ & 46.9 & 4.2 & 10 \\
\hline & & $1.2 \mathrm{~mm}$ & 42.4 & 4.05 & 10 \\
\hline & \multirow{3}{*}{ Speed } & $0.5 \mathrm{~mm}$ & 50.1 & 5.4 & 10 \\
\hline & & $0.8 \mathrm{~mm}$ & 49.2 & 4.5 & 10 \\
\hline & & $1.2 \mathrm{~mm}$ & 44.6 & 5.7 & 10 \\
\hline
\end{tabular}

TABLE (3) Microleakage scores of the test groups.

\begin{tabular}{|l|c|c|c|c|c|c|}
\hline & \multicolumn{3}{|c|}{ Conventional sintering } & \multicolumn{3}{c|}{ Speed sintering } \\
\cline { 2 - 7 } & $\begin{array}{c}0.5 \text { chamfer } \\
\text { width }\end{array}$ & $\begin{array}{c}0.8 \text { chamfer } \\
\text { width }\end{array}$ & $\begin{array}{c}1.2 \text { chamfer } \\
\text { width }\end{array}$ & $\begin{array}{c}0.5 \text { chamfer } \\
\text { width }\end{array}$ & $\begin{array}{c}0.8 \text { chamfer } \\
\text { width }\end{array}$ & $\begin{array}{c}1.2 \text { chamfer } \\
\text { width }\end{array}$ \\
\hline Mean (n=10) & 2.9 & 2.2 & 2 & 3.1 & 2.9 & 2 \\
\hline Median & 3 & 2 & 2 & 3 & 0.9 & 0.6 \\
\hline SD & 0.73 & 0.63 & 0.81 & 1 & 2 & 1 \\
\hline Minimum & 2 & 1 & 3 & 4 & 4 & 3 \\
\hline Maximum & 4 & 3 & & & 3 & 2 \\
\hline
\end{tabular}




\section{DISCUSSION}

Fabrication of CAD/CAM ceramic restorations includes several processing stages that could results in defects in the finished restorations. The result of this study revealed a significant difference on marginal discrepancy, internal adaptation and microleakage of monolithic zirconia crowns with respect to different chamfer widths tested while sintering protocols have no impact on both fit and microleakage of translucent zirconia. Therefore, the first part of null hypothesis was accepted while the second part was rejected.

In the current investigation, natural teeth were used to furnish a more clinically pertinent substrate with respect to preparation margin width, bonding protocol, and adhesive cementation that could affect the marginal fit, internal adaptation and microleakage evaluation values of monolithic zirconia crowns. Conventional milling of $\mathrm{ZrO}_{2}$ restorations is processed in one day, sintering is performed overnight, and the definitive manufacturing is performed the next day; while, with speed sintering process, milling, sintering and even veneering of the final restoration can be done in one day. ${ }^{31}$ in the current investigation, both marginal and internal fit of the study groups showed comparable values, that could be related to the strength and stability of the zirconia crowns that sintered in either conventional or speed sintering process. Speed sintering process have been emphasized to affect the biaxial flexural strength, grain size, and translucency of zirconia restorations; while, the effect of these modifications has not been assured on zirconia characteristics. The finding of this investigation was in accordance with a study conducted by Khaledi et $\mathrm{al}^{32}$ who stated that, speed sintering of zirconia crowns did not significantly alter the fit of the cemented restoration.

Evaluation of marginal discrepancy can be accomplished before or after cementation, with or without artificial ageing. In the present study, marginal gap was evaluated after cementation as it was reported that, evaluation of marginal gap before cementation does not reflect the real marginal fit in the oral cavity compared to measurement of discrepancy after cementation. Up till now, no universal standard exists on how to perform gap assessments, and clinically, no agreement has been reached on the acceptable precision of fit. Marginal discrepancy evaluation methods should be convenient, standardized, and reproducible. The four basic methods for marginal discrepancy evaluation include: cross-sectional, direct view, visual and tactile examination, and impression technique.

Previous researches recorded discrepancies below $100 \mu \mathrm{m}$ seem to be acceptable from clinical prospective point of view. ${ }^{33-35}$ In the current investigation, the values of gap measurements for all experimental groups were clinically acceptable except for the occlusal surface. Both marginal and axial gap results were nearly the same as those of the proposed cement space $(50 \mu \mathrm{m})$, however the discrepancies at the occlusal surface were markedly greater and could be explained by shrinkage of zirconia restorations following post-machining sintering. ${ }^{36}$

The limitations of CAD/CAM systems regarding to software designing restorations, hardware scanning equipment and the milling machine are possible short-comings in the $\mathrm{CAD} / \mathrm{CAM}$ technique. In addition, a size discrepancy of the cutting tools, tooth preparation geometry may cause decrease in occlusal adaptation values of CAD/CAM fabricated zirconia crown. ${ }^{36,37}$

Numerous factors could affect the shrinkage process of zirconia restorations, such as the material itself, density distribution, the compaction density, and the sintering process parameters. These factors was known as a central characteristic in a blank, that determines the local shrinkage, and subsequently the dimensional accuracy following final sintering process. $^{32}$

In the current study, a dye penetration technique was used in order to verify the margin 
microleakage. The technique is described as simple, dye penetration can be visualized more quickly and exposure time is shorter. It is worth mentioning that the number of dentinal tubules is small cervically, therefore compared to the rest of the preparation dye penetration at the tooth margin would be less harmful to the pulp. On the other hand, if dye penetration is at the tooth-cement interface, this indicates the presence of potential gap that could harbor bacteria and subsequently induces dental caries and periodontal problems ${ }^{38}$. In the current study, the greatest microleakage values were observed with $0.5 \mathrm{~mm}$ chamfer width group which might indicate potential harmful effects to the pulp.

Correlation values between misfit and microleakage were low because the discrepancy that occur at the tooth-restoration interface that is filled by the luting cement, partially affect the observed microleakage scores. The bond quality also of the cement could have a significant role in the microleakage as well, since the test cements were resin based cements based on Fick's first law of diffusion states that "the rate of material dissolution is independent of the exposed area (amount of luting agent)". ${ }^{39}$

It is well-documented that microleakage score had been affected by the type of tooth substrate. The differences in microleakage scores recorded for the tested groups were observed between crown margins bonded to enamel and margins bonded to dentin substrates as a result of difference in finish line width. Due to higher mineral content of enamel than those of dentin substrates, bonding to the enamel is more predictable and stable. Bonding studies to dentin substrates have reported deficiencies on the sealing capability to dentin compared to that of enamel..$^{40,41}$

A perfect marginal fit is impossible; there is no indirect restoration that could results in a precision fit. However, to fill the misfit between prepared tooth and indirect restoration, luting cements are used. The findings of this investigation support the concept that the rate of microleakage is influenced significantly by location and width of the preparation margin this could be explained by shrinkage by firing procedure of the zirconia crowns with decreased finish line width.

There were some limitations in the present study. The crowns were constructed under optimal and standardized conditions, however, in clinical practice, tooth preparation, impression and/ or cementation procedure all these factors could affect the clinical fit of the restoration. Additionally, no simulation of intraoral condition was assessed.

\section{CONCLUSIONS}

Marginal adaptation and internal fit of translucent monolithic zirconia crowns are influenced by width of the preparation margin while sintering protocol has little impact on adaptation.

\section{REFERENCES}

1. Ghodsi S, Jafarian Z. A Review on translucent zirconia. Eur J Prosthodont Restor Dent 2018; 26: 62-74.

2. Carrabba M, Keeling AJ, Aziz A, Vichi A, Fabian Fonzar $\mathrm{R}$, Wood D, et al. Translucent zirconia in the ceramic scenario for monolithic restorations: A flexural strength and translucency comparison test. J Dent 2017; 60: 70-76.

3. Malkondu O, Tinastepe N, Kazazoglu E. Influence of type of cement on the color and translucency of monolithic zirconia. J Prosthet Dent 2016; 116: 902-908.

4. Ebeid K, Wille S, Hamdy A, Salah T, El-Etreby A, Kern M. Effect of changes in sintering parameters on monolithic translucent zirconia. Dent Mater 2014; 30: e419-424.

5. Sorensen JA: The Lava system for CAD/CAM production of high strength precision fixed prosthodontics. Quintessence Dent Technol 2003; 26: 57-67.

6. Vagkopoulou T, Koutayas SO, Koidis P, et al: Zirconia in dentistry: Part 1. Discovering the nature of an upcoming bioceramic. Eur J Esthet Dent 2009; 4: 130-151

7. Denry I, Kelly R: State of the art of zirconia for dental application. Dent Mater 2008; 24: 299-307. 
8. Li RW, Chow TW, Matinlinna JP. Ceramic dental biomaterials and CAD/CAM technology: state of the art. J Prosthodont Res 2014; 58: 208-216.

9. Kelly JR, Denry I. Stabilized zirconia as a structural ceramic: an overview. Dent Mater 2008; 24: 289-298.

10. Attachoo S, Juntavee N. Role of sintered temperature and sintering time on spectral translucence of nano-crystal monolithic zirconia. J Clin Exp Dent 2019; 11: e146-e153.

11. Juntavee N, Attashu S. Effect of different sintering process on flexural strength of translucency monolithic zirconia. J Clin Exp Dent 2018; 10: e821-e830.

12. Juntavee N, Attashu S. Effect of sintering process on color parameters of nano-sized yttria partially stabilized tetragonal monolithic zirconia. J Clin Exp Dent 2018 ; 10: e794-e804.

13. Suya Prem Anand P, Arunachalam N, Vijayaraghavan L. Effect of grinding on subsurface modifications of pre-sintered zirconia under different cooling and lubrication conditions. J Mech Behav Biomed Mater 2018 ;86: 122-130.

14. Pereira GKR, Silvestri T, Amaral M, Rippe MP, Kleverlaan CJ, Valandro LF. Fatigue limit of polycrystalline zirconium oxide ceramics: Effect of grinding and low-temperature aging. J Mech Behav Biomed Mater 2016; 61: 45-54.

15. Sadan A, Blatz MB, Lang B. Clinical considerations for densely sintered alumina and zirconia restorations: Part 1 . Int J Periodontics Restorative Dent. 2005; 25: 213-219.

16. Inokoshi M, Zhang F, De Munck J, Minakuchi S, Naert I, Vleugels J, et al. Influence of sintering conditions on lowtemperature degradation of dental zirconia. Dent Mater 2014; 30: 669-678.

17. Ahmed WM, Abdallah MN, McCullagh AP, Wyatt CCL, Troczynski T, Carvalho RM. Marginal discrepancies of monolithic zirconia crowns: The influence of preparation designs and sintering techniques. J Prosthodont 2019; 28: 288-298.

18. Taha D, Spintzyk S, Sabet A, Wahsh M, Salah T. Assessment of marginal adaptation and fracture resistance of endocrown restorations utilizing different machinable blocks subjected to thermomechanical aging. J Esthet Restor Dent 2018;30(4):319-328.

19. Monaco C, Rosentritt M, Llukacej A, Baldissara P, Scotti R. Marginal adaptation, gap width, and fracture strength of teeth restored with different all-ceramic vs metal ceramic crown systems: An in vitro study. Eur J Prosthodont Restor Dent 2016; 24: 130-137.
20. Shembesh M, Ali A, Finkelman M, Weber HP, Zandparsa $\mathrm{R}$. An in vitro comparison of the marginal adaptation accuracy of CAD/CAM restorations using different impression systems. J Prosthodont 2017; 26: 581-586.

21. Sener I, Turker B, Valandro LF, Ozcan M. Marginal gap, cement thickness, and microleakage of 2 zirconia crown systems luted with glass ionomer and MDP-based cements. Gen Dent. 2014;62(2):67-70.

22. Korkut L, Cotert HS, Kurtulmus H. Marginal, internal fit and microleakage of zirconia infrastructures: an in-vitro study. Oper Dent. 2011; 36: 72-79.

23. Yüksel E, Zaimoğlu A. Influence of marginal fit and cement types on microleakage of all-ceramic crown systems. Braz Oral Res 2011; 25: 261-266.

24. Martínez-Rus F, Suárez MJ, Rivera B, Pradíes G. Influence of CAD/CAM systems and cement selection on marginal discrepancy of zirconia-based ceramic crowns. Am J Dent 2012; 25: 67-72.

25. Keshvad A, Hooshmand T, Asefzadeh F, Khalilinejad F, Alihemmati M, Van Noort R. Marginal gap, internal fit, and fracture load of leucite-reinforced ceramic inlays fabricated by CEREC inLab and hot-pressed techniques. J Prosthodont 2011; 20: 535-540.

26. Bindl A, Mörmann WH. Marginal and internal fit of all-ceramic CAD/CAM crown-copings on chamfer preparations. J Oral Rehabil 2005; 32: 441-447.

27. Euán R, Figueras-Álvarez O, Cabratosa-Termes J, Brufau-de Barberà M, Gomes-Azevedo S. Comparison of the marginal adaptation of zirconium dioxide crowns in preparations with two different finish lines. J Prosthodont 2012; 21: 291-295.

28. Beuer F, Aggstaller H, Richter J, Edelhoff D, Gernet W. Influence of preparation angle on marginal and internal fit of CAD/CAM-fabricated zirconia crown copings. Quintessence Int 2009; 40: 243-250.

29. Sener I, Turker B, Valandro LF, Ozcan M. Marginal gap, cement thickness, and microleakage of 2 zirconia crown systems luted with glass ionomer and MDP-based cements. Gen Dent 2014; 62: 67-70.

30. Sailer I, Makarov NA, Thoma DS, et al. All-ceramic or metal-ceramic tooth-supported fixed dental prostheses (FDPs)? A systematic review of the survival and complication rates. Part I: Single crowns (SCs) Dent Mater 2015; 31: 603-623. 
31. Ersoy NM, Aydoğdu HM, Değirmenci BÜ, Çökük N, Sevimay M. The effects of sintering temperature and duration on the flexural strength and grain size of zirconia. Acta Biomater Odontol Scand 2015; 1: 43-50.

32. Khaledi AAR, Vojdani M, Farzin M, Pirouzi S, Orandi S. The Effect of Sintering Time on the Marginal Fit of Zirconia Copings. J Prosthodont 2019; 28: e285-e289.

33. Euán R, Figueras-Álvarez O, Cabratosa-Termes J, OliverParra R. Marginal adaptation of zirconium dioxide copings: influence of the CAD/CAM system and the finish line design. J Prosthet Dent 2014; 112: 155-162.

34. Euán R, Figueras-Álvarez O, Cabratosa-Termes J, Brufaude Barberà $\mathrm{M}$, Gomes-Azevedo $\mathrm{S}$. Comparison of the marginal adaptation of zirconium dioxide crowns in preparations with two different finish lines. J Prosthodont 2012; 21:291-295.

35. Komine F, Iwai T, Kobayashi K, Matsumura H. Marginal and internal adaptation of zirconium dioxide ceramic copings and crowns with different finish line designs. Dent Mater J 2007; 26: 659-664.

36. Habib SR, Al Otaibi AK, Al Anazi TA, Al Anazi SM.
Comparison between five CAD/CAM systems for fit of zirconia copings. Quintessence Int 2018; 49: 437-444.

37. Guess PC, Vagkopoulou T, Zhang Y, Wolkewitz M, Strub JR. Marginal and internal fit of heat pressed versus CAD/ CAM fabricated all-ceramic onlays after exposure to thermo-mechanical fatigue. J Dent. 2014; 42: 199-209.

38. Gu X, Kern M. Marginal discrepancies and leakage of all-ceramic crowns: influence of luting agents and aging conditions. Int J Prosthodont. 2003;16:109-116.

39. Nicholson JW. The physics of water sorption by resinmodified glass-ionomer dental cements. J Mater Sci Mater Med 1997; 8: 691-695.

40. Liu Z, Zhao X, Zeng X, Xu S, Lu Y, Huang D. Effects of different resin adhesives on the microleakage in a new model with simulated subgingival condition and pulpal pressure. Dent Mater J 2018; 37: 761-767.

41. Onay EO, Yamanel K, Korkmaz-Ceyhan Y, Gulsahi K. Comparison of three adhesive systems in class II composite restorations in endodontically treated teeth: Influence of Er:YAG laser conditioning and gingival margin levels on microleakage. J Clin Exp Dent 2018; 10: e781-e788. 